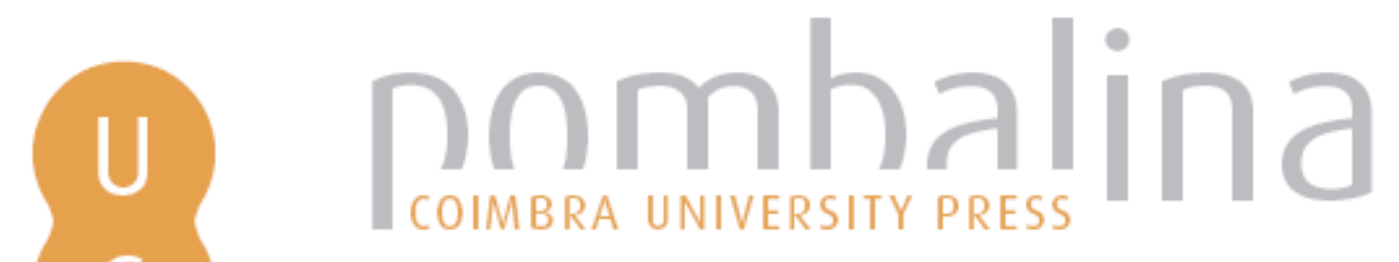

\title{
Consideraciones generales sobre la gastronomía en Aristófanes: aspectos léxicos
}

Autor(es): $\quad$ Rodríguez Moreno, Inmaculada

Publicado por: Imprensa da Universidade de Coimbra; Annablume

URL

persistente: URI:http://hdl.handle.net/10316.2/39619

DOI: $\quad$ DOI:https://doi.org/10.14195/978-989-26-1191-4_10

Accessed : $\quad$ 26-Apr-2023 15:25:00

A navegação consulta e descarregamento dos títulos inseridos nas Bibliotecas Digitais UC Digitalis, UC Pombalina e UC Impactum, pressupõem a aceitação plena e sem reservas dos Termos e Condições de Uso destas Bibliotecas Digitais, disponíveis em https://digitalis.uc.pt/pt-pt/termos.

Conforme exposto nos referidos Termos e Condições de Uso, o descarregamento de títulos de acesso restrito requer uma licença válida de autorização devendo o utilizador aceder ao(s) documento(s) a partir de um endereço de IP da instituição detentora da supramencionada licença.

Ao utilizador é apenas permitido o descarregamento para uso pessoal, pelo que o emprego do(s) título(s) descarregado(s) para outro fim, designadamente comercial, carece de autorização do respetivo autor ou editor da obra.

Na medida em que todas as obras da UC Digitalis se encontram protegidas pelo Código do Direito de Autor e Direitos Conexos e demais legislação aplicável, toda a cópia, parcial ou total, deste documento, nos casos em que é legalmente admitida, deverá conter ou fazer-se acompanhar por este aviso. 


\title{
ConSIDERACIONES gENERALES SOBRE LA GASTRONOMÍA en Aristófanes. Aspectos léxicos General considerations about gastronomy in Aristophanes. Lexical aspects
}

\author{
Inmaculada Rodríguez Moreno \\ Universidad de Cádiz \\ Facultad de Filosofía y Letras \\ inma.rodriguez@uca.es
}

El presente estudio ofrece un sucinto análisis del léxico sobre gastronomía utilizado por Aristófanes, representante principal de la comedia griega antigua. Nuestro objetivo consiste en mostrar una imagen de la vida cotidiana en la Atenas del siglo $\mathrm{V}$ a. C., a través de las comedias conservadas del eminente comediógrafo.

Palabras clave: gastronomía, Aristófanes, comedia griega.

The present study provides a succinct analysis of the Aristophanes' lexicon about gastronomy, the most representative author of the ancient Greek comedy. Our objective is to showan image of daily life in the Athens of the fifth century B.C., through the surviving comedies of this eminent playwright.

Keywords: gastronomy, Aristophanes, Greek comedy.

Pese a las referencias y descripciones que nos ofrecen la épica y la lírica, la fuente principal para rastrear la gastronomía griega reside en la comedia antigua, con Aristófanes a la cabeza, quien hace gala de una gran destreza pictórica a la hora de dibujar magníficos bodegones mediante el léxico. Los aspectos ilustrados son muy variados, desde el ir a comprar al mercado, los guisos o los horarios de comida hasta los utensilios propios de la cocina. A todo ello hay que añadir, además, diferentes tipos de panes y pasteles, tanto los que se consumían en cualquier ocasión como los destinados a un determinado ritual o festividad religiosa ${ }^{1}$. Así pues, el objetivo del presente estudio consiste en aportar una idea aproximada de las distintas facetas del arte culinario a través de las obras y fragmentos conservados del mayor representante de la comedia ática.

El verbo genérico que expresa el acto de comer es $\dot{\varepsilon} \sigma \theta i \omega^{2}$, en tanto que sus compuestos designan modalidades más concretas, como $\kappa \alpha \tau \varepsilon \sigma \theta i ́ \omega$, cuyo

\footnotetext{
${ }^{1}$ Cf. Wilkins 1993: 66-74; Wilkins 1996: 46-56; Wilkins 2000.

${ }^{2}$ Pax 23; 33; 48; 449; 643; 1116; 1166; 1284; 1285, Ec. 358; Pl. 931; 1123; Eq. 606; 707; 937; 939; 1106; 1181; 1294; Ach. 79; 797; 799; V. 194; 610; 1367; Av. 76.
} 
preverbio especifica hacerlo con los cinco sentidos puestos en la comida, con la cabeza hacia abajo, sin reparar en todo cuanto acontece alrededor, de donde se entiende comer rápidamente o con ansia. En el siguiente ejemplo,

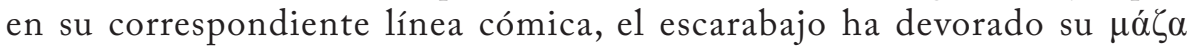
(eufemismo por excremento) en un santiamén, sin saborear ni disfrutar de su "delicioso" manjar':

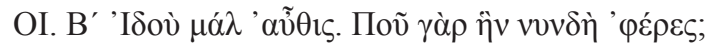

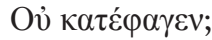

Esclavo segundo: "Ahí lo tienes también. ¿̇Pero dónde está la que (sc. torta de cebada) le trajiste hace poco? No se la ba comido ya?".

$\Pi \alpha \rho \varepsilon \sigma \theta i ́ \omega$ indica consumir alimentos fuera de los horarios establecidos, es decir, picar entre horas, bien sea como tentempié, por gula o simplemente para calmar el hambre 4 . Es entonces cuando se ingieren frutos secos, golosinas o aperitivos. Relacionado con estas viandas está $\tau \rho \omega ́ \gamma \omega$, verbo onomatopéyico que emula el peculiar ruido que se realiza al masticar:

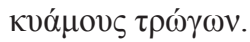

"Comiendo habas"s.

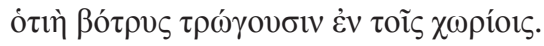

"porque se comen las uvas en los campos".

De igual modo, su compuesto $\pi \alpha \rho \alpha \tau \rho \omega ́ \gamma \omega$ insinúa comer estos piscolabis, pero hasta el final, de acuerdo con el preverbio ${ }^{7}$ :

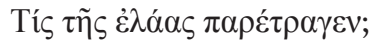

"¿Quién se ha zampado las aceitunas?”.

Por otro lado, con $\dot{\varepsilon} \xi \varepsilon \sigma \theta \dot{i} \omega^{8}$ se alude a un cierto grado de voracidad e que va más allá de lo que se pueda manifestar con el verbo en su forma simple, llegando a tragar o engullir el alimento como si se tratara de una píldora $(\kappa \alpha \tau \alpha \beta \rho \circ \chi \theta i \zeta \omega)^{9}$ :

\footnotetext{
${ }^{3} \operatorname{Pax} 5$.

${ }^{4}$ Eq. 1026.

${ }^{5}$ Lys. 537.

${ }^{6}$ Eq. 1077.

${ }^{7}$ Ra. 988.

${ }^{8}$ Eq. 1030-1032.

${ }^{9}$ Eq. 356-358.
} 


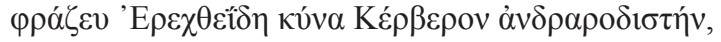

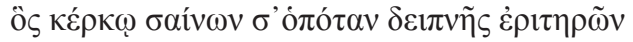

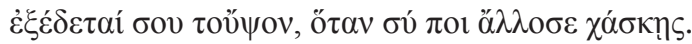

"Observa, hijo de Erecteo, al can Cerbero, el esclavizador, que, acechándote, meneando el rabo, mientras cenas, devorará tu comida, cuando te retires a otra parte".

Otro compuesto es $\dot{\alpha} \pi \varepsilon \sigma \theta i ́ \omega$, comer hasta el final convulsivamente. Tal es la manera de actuar de una corneja en su afán por morder los dedos de Pistetero, según se colige de este fragmento no exento de comicidad ${ }^{10}$ :

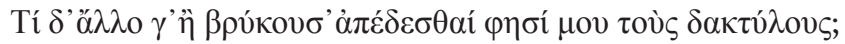
"QQué otra cosa dice sino que, por roer, me terminará de comer los dedos?".

Como resultado de lo anterior, se llega a la modalidad que connota

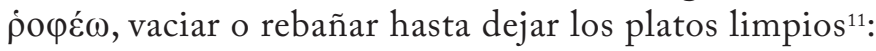

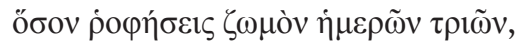

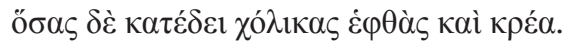

"Cuánta salsa tragarás en tres días" ${ }^{12}$ cuánta carne y entrañas cocidas devorarás!"

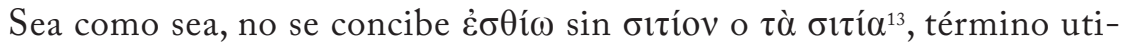
lizado para todo alimento preparado por las manos del hombre ${ }^{14}$, dado que deriva de $\sigma i ̃ \tau \zeta$, , es decir, el grano de trigo con el cual se hace el pan. De ahí

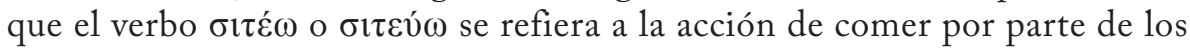
seres humanos ${ }^{15}$ :

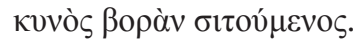

"alimentándose de comida de perro".

Distinto es cuando se trata de dar de comer a otro. Precisamente, para los niños más pequeños, es necesario desmenuzar la comida en trozos minúsculos o triturarla hasta convertirla en papilla, para luego poner los pedazos en la boca, acto que denota el verbo $\psi \omega \mu$ í̧ $\omega$, de donde $\psi \omega \mu$ ó, , bocado, tajada ${ }^{16}$ :

\footnotetext{
${ }^{10}$ Av. 26.

${ }^{11}$ Pax 716-717.

${ }^{12}$ Los días habituales que suelen durar las fiestas.

${ }^{13}$ Eq. 709.

${ }^{14}$ Pax 723.

${ }^{15}$ Eq. 416.

16 Th. 692.
} 


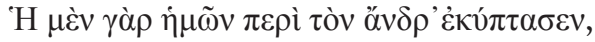

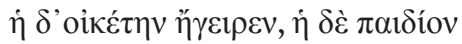

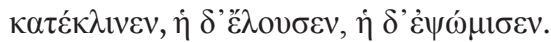

"Entre nosotras una estaba acostada con el marido, otra despertaba al criado, otra acostaba al niño, otra lo lavaba, y otra le daba de comer".

En definitiva, la consecuencia inmediata de comer en todas sus categorías es alimentar, $\tau \rho \varepsilon ́ \varphi \omega$, bien se trate de los hijos o de uno mismo ${ }^{17}$ :

oủ ' '

"Tú ya no alimentarás a los hijos que tienes, sino tu mujer".

Respecto a la ingestión de líquidos, el verbo simple es $\pi$ ív $\omega^{18}$, mientras que beber acompañándose de un aperitivo o beber encima de la comida es غ̇ंı $\pi \dot{v} v \omega$ :

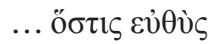

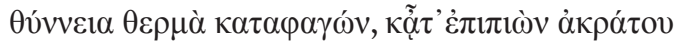

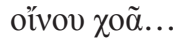

“... quien (sc. yo) me trago una ración calentita de atún y me bebo encima una jarra de vino puro..."

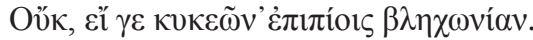

No, si no bebes encima una infusión de poleo"20.

Por tanto, en función del preverbio, $\pi \rho 0 \pi$ ív $\omega^{21}$ significa "beber antes o el

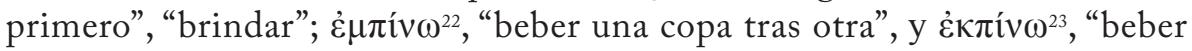
hasta el fondo", "apurar el vaso":

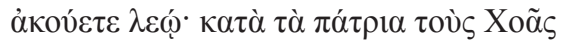

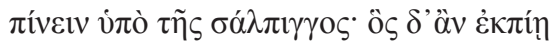

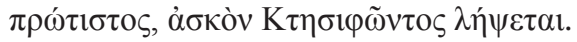

"Oid..., gentes,... según la costumbre de los antepasados, bebed al son de la trompeta. Y quien la apure primero, recibirá un odre como la barriga de Ctesifonte".

${ }^{17}$ Ec. 461.

${ }^{18}$ Cf. por ejemplo, Th. 630; Ec. 132; 133; Ra. 1150; Nu. 1358; 1382; Pl. 972; 1184; Eq. 49; 888; Ach. 79; V. 575; 1198.

${ }^{19}$ Eq. 353-355.

${ }^{20}$ Pax 712.

${ }^{21}$ Th. 631.

${ }^{22}$ Pax 1146; 1156.

${ }_{23}$ Ach. 1000-1003. 
Sin embargo, el acto intermedio entre comer y beber es $\lambda \varepsilon^{i} \chi \omega$, "lamer", para lo cual no se utilizan los dientes, como hacen las personas que, al carecer de ellos, no pueden masticar alimentos sólidos. De esta forma, del siguiente pasaje se deduce que la textura de la ambrosía de los dioses era espesa ${ }^{24}$ :

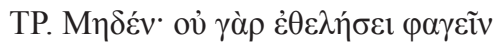

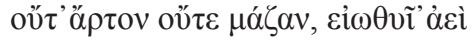

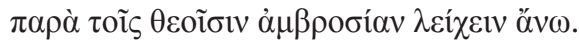

Trigeo: "Nada, pues no querrá comer pan candeal ni de cebada, ya que está acostumbrada a lamer arriba con los dioses la ambrosía".

Por otra parte, una vertiente fundamental y propia de la gastronomía apunta a las diversas maneras de cocinar los alimentos. Éstas son descritas mediante verbos onomatopéyicos construidos a partir del sonido que se percibe en el momento en que aquéllos se encuentran al fuego: $\check{\psi} \psi \omega^{25}$ ("guisar" o "cocinar carnes o pescados en líquido o en su jugo", "estofar"), $\beta \rho \alpha ́ \sigma \sigma \omega / \beta \rho \alpha ́ \tau \tau \omega^{26}$ ("hervir"), $\pi \dot{\varepsilon} \sigma \sigma \omega^{27}$ ("hornear"), ỏ $\pi \tau \alpha \dot{\alpha} \omega^{28}$ ("asar"), y $\varphi \rho v ́ \gamma \omega^{29}$ ("tostar"), el cual imita en su pronunciación el ruido de la carne o del pescado mientras se está

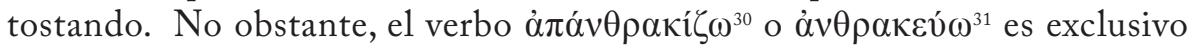
para la carne cuando se asa en una parrilla de carbón, pues consiste en un derivado de $\alpha 2 v \theta \rho \alpha \xi$, carbón ${ }^{32}$. En consecuencia, $\dot{z} \pi \alpha \nu \theta \rho \alpha \kappa i ́ \delta \varepsilon \varsigma^{33}$ se utiliza para el pescado asado sobre carbones:

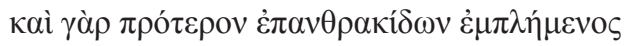

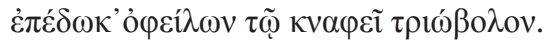

"Otra vez que me harté de pescado asado (sobre carbones), pagué tres óbolos al cardador".

En cuanto a los alimentos y manjares mencionados en las comedias aristofánicas, habría que comenzar por aquellos vocablos empleados para el pan, según el material de su preparación. Así pues, $\mu \alpha \dot{\zeta} \zeta \alpha^{34}$, de $\mu \alpha \alpha \sigma \sigma \omega$ ("amasar"),

${ }^{24} \operatorname{Pax} 852-854$.

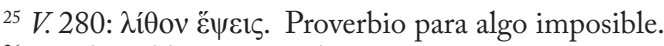

${ }^{26}$ Ra. 510; 558; Ach. 1005.

${ }^{27} \mathrm{Pl} .1136 ; 1142$.

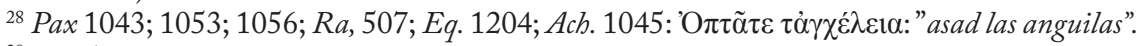

${ }^{29}$ Ra. 511.

${ }^{30}$ Ra. 505.

${ }^{31}$ Lys. 340.

${ }^{32}$ Frag. 68.

${ }^{33}$ V. 1127.

${ }^{34}$ Ra. 1073; Ec. 851; Pl. 192; Ach. 732; 835, $\mu \tilde{\alpha} \delta \delta \alpha$ en el dialecto megarense. Cf. García 2001: 95-98. Jasny 1950: 227-253. 
denomina un tipo de pan o torta elaborado con harina de cebada ${ }^{35}$, la cual le da su particular color oscuro ${ }^{36}$. Éste era mayoritariamente consumido por los

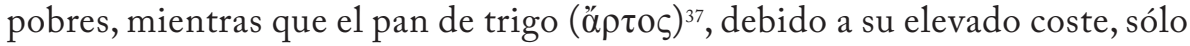
se lo podían permitir quienes tuvieran cierto poder adquisitivo. Aristófanes dice que su precio es de diez óbolos, cuando una panadera se lamenta de la pérdida de sus existencias ${ }^{38}$ :

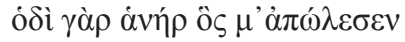

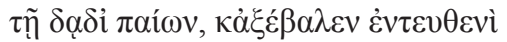

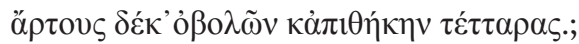

"Este hombre es quien me ha arruinado pegándome con la antorcha, y me ha tirado por el suelo panes de diez óbolos y un resto de serie por valor de cuatro".

Por supuesto, habida cuenta de su valor, no cabe duda de que su exquisito sabor y calidad son superiores a los de la $\mu \alpha \dot{\zeta} \alpha^{39}$ :

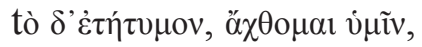

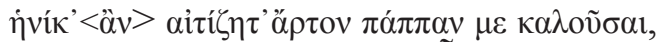

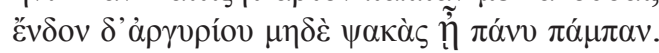

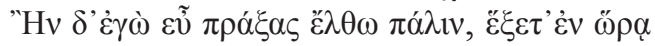

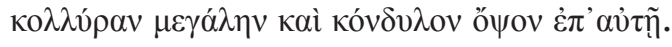

"La verdad es que sufro por vosotras, cuando me pedís pan blanco llamándome papá, $y$ dentro de casa no hay siquiera una migaja de plata. Si regreso bien de mi empresa, tendréis a tiempo una gran hogaza y salsa de puñetazo sobre ella".

En este último ejemplo nuestro cómico cita una variedad panificadora

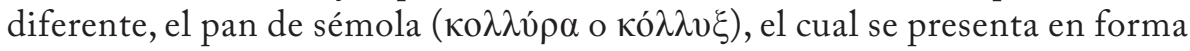
enroscada. En el pasaje Trigeo se siente afligido porque sus hijas desean $\alpha$ $\tau o \varsigma$ y él sólo puede ofrecerles $\mu \alpha \dot{\zeta} \alpha$, por lo que va a ser víctima de una

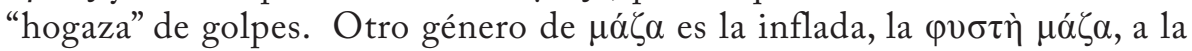
manera de tiernos bollitos ${ }^{40}$ :

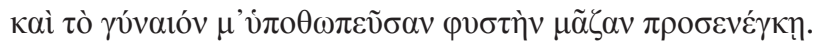

"Y mi mujer, dándome coba, me entrega un tierno bollito".

\footnotetext{
${ }^{35}$ Moritz 1958.

${ }^{36}$ Amouretti:1986; Braun 1995: 29-30.

${ }^{37}$ Lys. 1206; Nu. 1383; Pl. 190; 1135, Eq. 282.

${ }^{38}$ V.1389-1391.

${ }^{39}$ Pax 119-123.

${ }^{40}$ V. 610 .
} 
También, en las comedias de Aristófanes, se aplica la voz $\mu \alpha \zeta i ́ \sigma \kappa \eta$, diminutivo de $\mu \alpha ́ \zeta \alpha$, a una torta pequeña de harina de cebada ${ }^{41}$ :

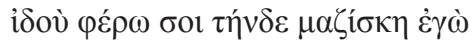

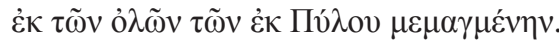

"Mira, yo te traigo esta torta amasada con los granos de cebada de Pilo".

En lo concerniente a la repostería, nuestro cómico ofrece todo un catálogo léxico, en el que habría que distinguir entre los pasteles destinados a ser ofrendas a la divinidad ${ }^{42}$ y los consumidos en cualquier ocasión. Dentro de los primeros está el $\pi$ ó $\pi \alpha v o v$, ofrenda dirigida a Deméter y Perséfone, con harina de cebada, miel y queso ${ }^{43}$ :

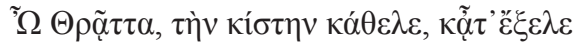

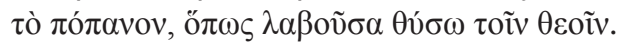

"Deja, tracia, la cesta en el suelo y saca de ella una torta para cogerla y ofrecerla en sacrificio a las dos diosas".

Además, destacan el $\psi \alpha \_\sigma \tau o ́ v^{44}$ a Hera o los héroes, con harina de cebada,

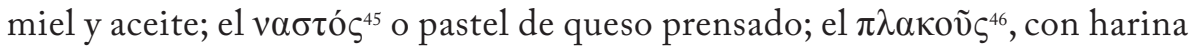
de sésamo, miel y queso; el $\sigma \eta \sigma \alpha \mu o \varepsilon i \varsigma^{47}$ o bola de sésamo tostado, aceite y miel, ambos para las bodas, y la $\mu \varepsilon \lambda ı \tau o \tilde{v} \tau \tau \alpha^{48}$, torta remojada en miel, con agua, harina de cebada y aceite, característica de las ceremonias fúnebres:

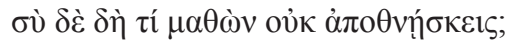

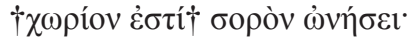

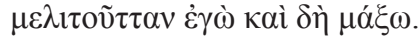

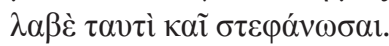

"Y tú ¿por qué no te mueres? Sitio hay, cómprate un ataúd, que yo voy a amasar la torta. Toma esto y póntelo de corona".

\footnotetext{
${ }^{41}$ Eq. 1166; 1105.

${ }^{42}$ Para los pasteles como ofrendas a la divinidad, cf. Blumfield 1997: 147-172; García 2014: 905-910.

${ }^{43}$ Th. 285-286; Pl. 660. Cf. García 2001: 389.

44. Pl. 138. Cf. García 2001: 389.

${ }^{45} \mathrm{Pl} .1142$.

${ }^{46}$ Pl, 191; 995; 1126, en honor de Hermes. Cf. etiam Eq. 1191; 1219; Ach. 1092.

${ }^{47}$ Ach. 1092. Cf. García 2001: 390.

${ }^{48}$ Lys. 601.
} 
En el segundo grupo de pasteles se encuentran el $\alpha{ }^{\prime} \mu \nu \lambda \mathrm{o} \varsigma^{49} \mathrm{o}$ torta para

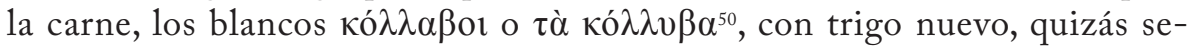
mejantes a los colines, y la oivoṽ $\tau \alpha$, especie de bizcocho borracho en vino, con harina, miel, agua y aceite ${ }^{51}$ :

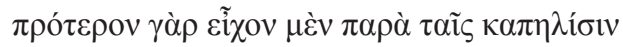

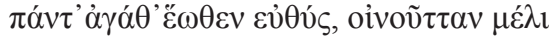

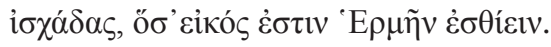

"Yo antes obtenía de las tenderas todos los alimentos buenos al instante desde temprano: bizcocho borracho, miel, higos, todo cuanto normalmente come Hermes".

Dejando a un lado los dulces, la dieta griega exhibida por Aristófanes no dista mucho de la llamada mediterránea, en la que figura la carne $(\kappa \rho \varepsilon ́ \alpha \varsigma)^{52}$, en todas sus variedades comestibles, la cual solía ser muy cara, por lo que sólo era consumida en ocasiones excepcionales, como en un sacrificio a la divinidad ${ }^{53}$ :

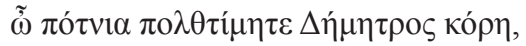

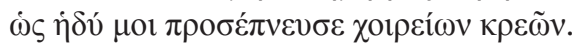

"Oh soberana, muy venerada hija de Deméter!

¡Qué dulce olor a carne de cerdo sopla hacia mí!”.

En cambio, los embutidos ( $\chi 0 \rho \delta \eta ́)$ resultaban más asequibles a los bolsillos, al igual que toda clase de verduras y legumbres ${ }^{54}$ y los pescados

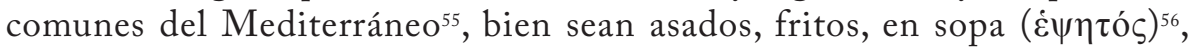
ahumados $(\tau \alpha \dot{\rho} \rho \imath \chi \varsigma)^{57}$, o acompañados de salsas como la $\sigma \kappa о \rho о \delta \alpha ́ \lambda \mu \eta$, con ajo y salmuera ${ }^{58}$, o la de silfio ${ }^{59}$ con queso, aceite y vinagre ${ }^{60}$ :

${ }^{49}$ Pax 1195; Ach. 1093. Cf. García 2001: 381.

${ }^{50}$ Pax 1196; Ra. 507. Cf. García 2001: 92.

${ }^{51} \mathrm{Pl} .1120-1122$.

${ }^{52}$ Eq. 282; 420; Ach. 1051. Cf. García 2001: 217-240.

${ }^{53}$ Ra., 336-337.

${ }^{54}$ Cf. García 2001: 66-67.

${ }^{55}$ Davidson 19812; García 1997: 279-285.

${ }^{56} \mathrm{~V} ., 679$.

${ }^{57}$ Ach., 967; V., 491.

${ }^{58}$ Eq., 199; 1095.

${ }^{59}$ Planta que se utilizaba como condimento, hoy en día desaparecida, que era muy apreciada en la Antigüedad. En latín laserpicio. Cf. Salza 2005: 163; Bonacelli 1924; Feuardent 1948: 27-32; Rainaud 1911: 1337-1340; Sterier 1927: 103-114.

${ }^{60}$ Av. 531-534. 


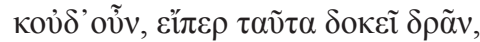
ỏ $\tau \tau \eta \sigma \alpha ́ \mu \varepsilon v o r ~ \pi \alpha \rho \alpha ́ \theta \varepsilon v \theta$ ’’ $\mu \tilde{\alpha} \varsigma$,

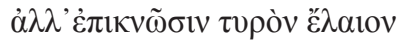

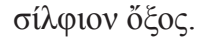

"Por tanto, si está bien actuar así, no se contentan con asaros, sino que os hacen picadillo con queso, aceite, silfio y vinagre”.

Un pescado que el Ateniense resalta por su delicioso sabor es la murena

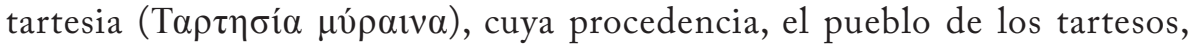
se ubica en el suroeste de la península ibérica, o sea, en los confines más occidentales del mundo conocido, y quizás por ello, de acuerdo con Javier de $\mathrm{Hoz}^{61}$, se pueda asociar con el Tártaro ${ }^{62}$ :

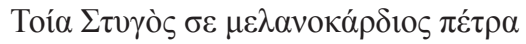

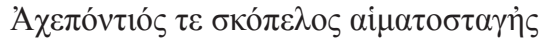

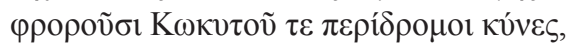

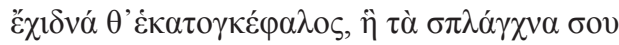

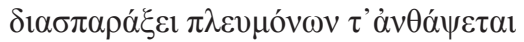

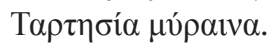

"Así la piedra de corazón negro de la Estige y el acantilado ensangrentado del Aqueronte te vigilan y los perros que corren alrededor del Cocito y Equidna de cien cabezas, la cual te desgarrará tus entrañas, y se cogerá de tus pulmones la murena tartesia".

Aristófanes, en cuanto a platos más elaborados, menciona el $\mu v \tau \tau \omega \tau \tau^{6} \varsigma^{63}$,

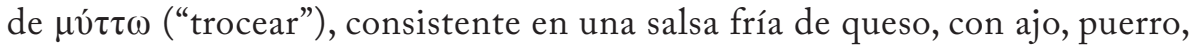
vinagre, aceite, huevo y miel ${ }^{64}$; las hojas de higuera asadas con grasa de buey

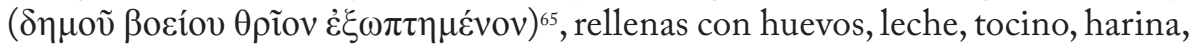
miel, queso o lonchas de pescado ${ }^{66}$, y los famosos purés (हैं $\tau o \varsigma$ ), como el de

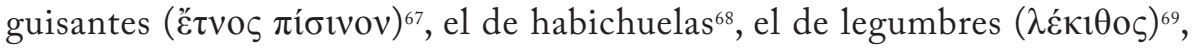
y el de lentejas $(\varphi \alpha \kappa \tilde{\eta})^{70}$, tan frecuentes en las mesas de los pobres ${ }^{71}$ :

${ }^{61}$ Hoz 2011: 225. Cf. Stanford 1963: ad loc.; Dover 1993: ad loc.

${ }^{62}$ Ra. 470-475.

${ }^{63}$ Eq. 771; Pax 273; Ach. 174. Cf. García 2001: 371-372.

${ }^{64}$ Cf. García 2007: 236-288.

${ }^{65}$ Eq. 954.

${ }^{66}$ Ach. 1001-1002.

${ }^{67}$ Lys. 1061; Eq. 1171; Ach. 246.

${ }^{68}$ Sobre el modo de preparación, Apicio propone varios modos: 3.15

${ }^{69}$ Lys. 561.

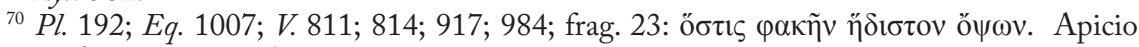
recomienda tres recetas: 5.2 .

${ }^{71}$ Pl. 1004-1005. 


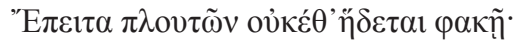

$\pi \rho o ̀ ~ \tau o \tilde{~} \delta$ 'vं

"Desde que es rico ya no le gusta el puré de lentejas, y antes, por la pobreza, comía de todo hasta el final".

Ahora bien, el diestro pintor de este arte culinario es el $\mu \alpha ́ \gamma \varepsilon ı \rho \varsigma^{72}$, sustantivo procedente de $\mu \alpha ́ \sigma \sigma \omega$ ("amasar"), actividad esencial relacionada con la fabricación de pan, el alimento más básico ${ }^{73}$. Sus funciones, designadas como $\mu \alpha \gamma \varepsilon \imath \iota_{\kappa} \alpha^{74} \mathrm{o} \mu \alpha \gamma \varepsilon \iota \rho \iota \tilde{\omega} \zeta^{75}$, eran desempeñadas sobre todo por mujeres o esclavos, hasta que en el siglo IV a. C. aparecen los primeros artífices de la cocina, los cuales llegaron a agruparse en gremios. Algunos, incluso, a raíz de su prestigio, escribieron manuales de cocina, como es el caso de "Tearión, el pastelero, Miteco, autor de un tratado sobre la cocina siciliana, y Sarambo, el comerciante de vinos, tres eminentes especialistas en pastelería, cocina y vinos"76. En la Comedia Media el cocinero pasa a formar parte del elenco de personajes, luciendo una gratuita charlatanería y un carácter embaucador.

$\mathrm{Su}$ taller era la cocina, ï $\pi v o \varsigma$, término que en principio alude al horno, objeto esencial de este recinto ${ }^{77}$. Allí tampoco deben faltar el horno abierto $(\dot{\varepsilon} \sigma \chi \alpha ́ \rho \alpha)^{78}$, la despensa $(\sigma 0 \pi u ́ \eta)^{79}$, y, lógicamente, los imprescindibles cacharros

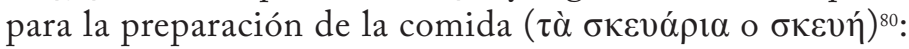

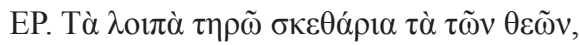

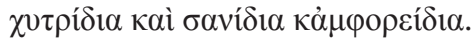

Hermes: "Vigilo los demás cacharros de los dioses, los pucherillos, las tablillas y las anforitas".

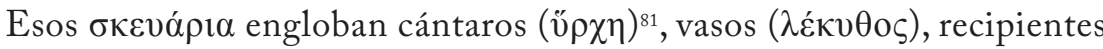

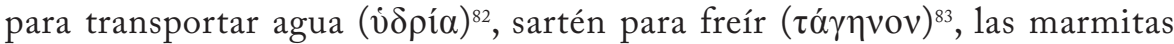
u ollas $(\chi v i \tau \rho)^{84}$, el cucharón o paleta para remover la comida $(\tau o \rho v ́ v \eta)^{85}$, el

${ }^{72}$ Ra. 517; Eq., 418. Cf. Berthiaume 1982.

${ }^{73}$ Cf. Daremberg.-Saglio 1962-3: s. v. coquus.

${ }^{74}$ Eq. 216. Frag. 138.

${ }^{75}$ Pax 1017; Ach. 1015.

${ }^{76} \mathrm{Pl}$. Grg. 518 b.

77 Thédenat 1896: 1420-1421.

${ }^{78}$ V. 937.

${ }^{79}$ Eq. 1296.

${ }^{80}$ Pax 201-202.

${ }^{81}$ V. 676

${ }^{82}$ V. 926.

${ }^{83}$ Eq. 929.

${ }^{84}$ Ra. 983; Pl. 673; 812; 1203-1206; Eq. 1176; Ach. 284; V. 904; 937; Av. 78; 358; Ec. 744; Ach. 463; Av. 78; 79.

${ }^{85}$ Eq. 984. 
almirez $(\theta v \varepsilon i ́ \alpha)^{86}$, el rallador de queso $(\tau v \rho о \kappa v \eta ́ \sigma \tau \iota \varsigma)^{87}$, la mano de mortero

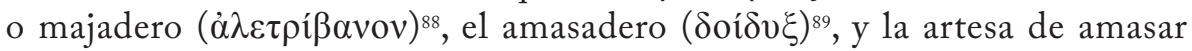
$(\kappa \alpha ́ \rho \delta о \pi о \varsigma)^{90}$.

En la mesa han de estar presentes, además de la cuchara y el cuchillo

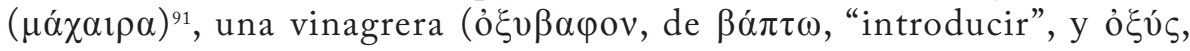

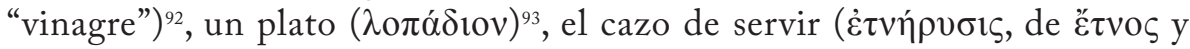

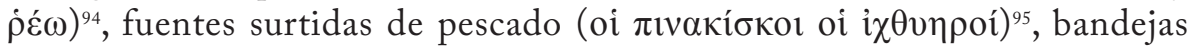
$(\pi \text { ív } \alpha \kappa \varepsilon \varsigma)^{96}$, y escudillas o cuencos $(\tau \rho v ́ \beta \lambda 1 \alpha)^{97}$. Sin embargo, los griegos no conocen el tenedor, sino que en su lugar empleaban la $\mu \alpha \dot{\alpha} \zeta \alpha$ o el ö $\rho \tau$ o como soporte, o la $\mu v \sigma \tau i ́ \lambda \eta$, es decir, cortezas de pan sin miga a modo de cuchara ${ }^{98}$ :

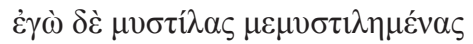

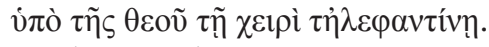

"Yo (sc. traigo) cortezas de pan con la miga quitada por la mano de marfil de la diosa".

De igual manera, la $\dot{\varepsilon} \lambda \alpha \tau \eta ́ \rho$, una torta plana y ancha, podía servir de plato $^{99}$ :

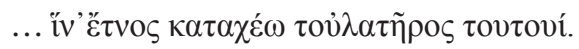

"... para echar puré en esta torta".

Los griegos no solían usar servilletas, sino aguamaniles, o bien se limpiaban los dedos con bolitas de migas de pan, $\dot{\alpha} \pi \circ \mu \alpha \gamma \delta \alpha \lambda i \alpha^{100}$ (de $\dot{\alpha} \pi \circ \mu \alpha ́ \sigma \sigma \omega)^{101}$, como se puede observar en el pasaje siguiente, en el que el personaje, para presumir de su riqueza, se retira la suciedad de sus dedos con el pan más caro, el $\alpha \chi \chi \imath \lambda \lambda \varepsilon$ ío $^{102}$, torta hecha con harina de cebada de la mayor calidad ${ }^{103}$ :

\footnotetext{
${ }^{86} \mathrm{Pax} 228 ; \mathrm{Nu} .675$.

${ }^{87}$ Frag. 7; V. 937; 963.

${ }^{88}$ Pax 259; 266; 269.

${ }^{89}$ Pax 288; 295. Eq. 984; V., 936.

${ }^{90} \mathrm{Nu} .673-678 ; 1251$.

${ }^{91}$ Pax 948.

${ }_{92}$ Pl, 812; Av. 361; Ach. 34. Cf. García 2001: 337-338.

${ }^{93} \mathrm{Pl} .812$

${ }^{94}$ Ach. 245

${ }^{95}$ Pl. 813-814.

${ }^{96} \mathrm{Pl} .996$.

${ }^{97}$ Ra. 985; Eq. 905; Ach. 277; V. 936; Av. 77; 361.

${ }^{98}$ Eq. 1168-1169.

${ }^{99}$ Ach. 246.

${ }^{100}$ Eq. 415.

${ }^{101}$ Eq. 819.

${ }^{102}$ Ath. 3. 11. 4 y sgtes. Cf. Thphr. HP. 8. 10.2.

${ }^{103}$ Eq. 819.
} 


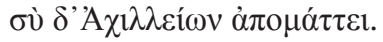

"Tú te limpias los dedos con el pan de lujo".

Como utensilios para el vino, se nombran las tinajas grandes de barro donde se almacenaba el preciado líquido ( $\pi \dot{i} \theta$ ov otvov $)^{104}$, y el colador de malla o una tela trenzada $(\tau \rho v \gamma o i ́ \pi \circ \varsigma)^{105}$, con los que se recogían las heces $(\tau \rho v ́ \gamma \eta)^{106:}$

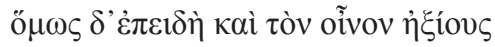

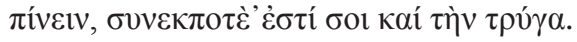

"Sin embargo, dado que has tenido a bien beber el vino, ahora puedes apurar también las heces".

El vino es el fiel compañero de la comida y juntos se convierten en los amigos de la fiesta, sin olvidar la música y los juegos ${ }^{107}$. No era habitual consumirlo puro, sino que se rebajaba y, para ello, previamente se vertía en

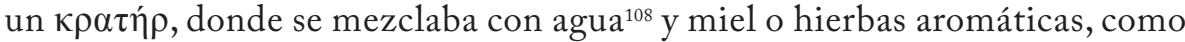
tomillo, menta o canela. Luego, esta mezcla, el aqua mulsa de los romanos ${ }^{109}$, se servía en copas normales ( $\pi$ otท́pıov) ${ }^{110}$ o en una copa ancha denominada $\kappa v ́ \lambda 1 \xi^{111}$, propia de ocasiones solemnes o festivas, con ayuda de unos cacillos. En cambio, cuando se quería llevar vino fuera de casa, se empleaban odres $(\dot{\alpha} \sigma \kappa o ́ \varsigma)^{112}$ de piel de cabra o de cerdo.

Por último, los horarios de las comidas principales se distribuyen en tres momentos del día. En el espacio comprendido entre las 7 y las 9 a. m.

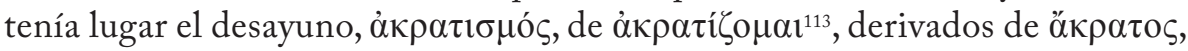
"puro", dado que era la única ocasión en que se tomaba como bebida excitante

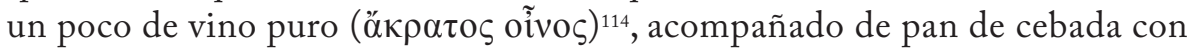
otros alimentos ${ }^{115}$ :

\footnotetext{
${ }^{104} \operatorname{Pax} 703$.

105 Pax 539.

106 Pl. 1084-1085.

${ }^{107}$ Bowie 1995: 113-125.

${ }^{108}$ Ec. 841.

${ }^{109}$ Dsc. 5. 9; Gal. 6. 274 K; Plin. 14. 113; Colum. 12. 12; Pallad. 8. 7. Cf. García 2001: 316.

${ }^{110}$ Eq. 120; 122.

${ }^{111}$ Lys. 234.

${ }_{112}$ Ach. $1225 ; 1230$.

${ }^{113}$ Pl. 295.

${ }^{114}$ Eq. 105; Ach. 75; 1229.

${ }^{115}$ Ec. 308.
} 


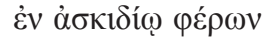

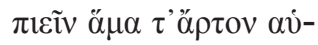

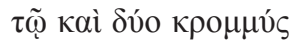

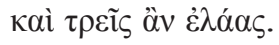

"Trayendo su botita de vino para beber, con pan, dos cebollas y tres aceitunas".

Después, entre las 11 ó 12 a. m., se efectuaba el almuerzo (ő $\rho 1 \sigma \tau o v$, de

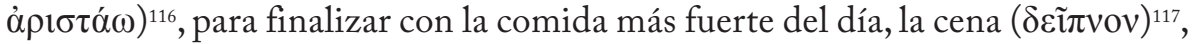
si bien en medio podía situarse la merienda $(\dot{\varepsilon} \sigma \pi \varepsilon \dot{\varepsilon} \rho \iota \mu \alpha)$. Generalmente la cena consistía en platos más elaborados ${ }^{118}$ :

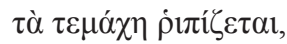

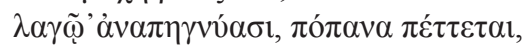

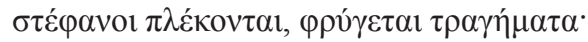

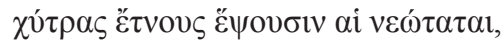

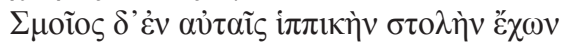

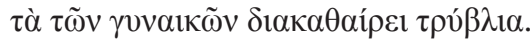

"Ya están hechas vuelta y vuelta las lonchas de pescado, se clavan las liebres, se hornean tortas, se trenzan coronas y se frien los pastelillos, las jóvenes guisan las ollas de puré, y Esmeo, con su uniforme de caballería entre ellas, friega los platos de las mujeres".

Con ella comenzaban los banquetes, antes de pasar a la reunión de

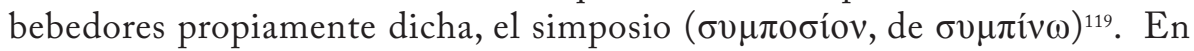
él, los comensales $(\sigma \nu \mu \pi o ́ \tau \alpha \imath)^{120}$ saboreaban distintas variedades de vino: blanco (oĩvo $\lambda \varepsilon v \kappa o ́ \varsigma$ ), oscuro (oĩvo $\varsigma \mu \varepsilon ́ \lambda \alpha \varsigma$ ), o el nuevo recién vendimiado, llamado $\tau \rho v \gamma o ́ \varsigma^{121}$. De los vinos con denominación de origen, Aristófanes

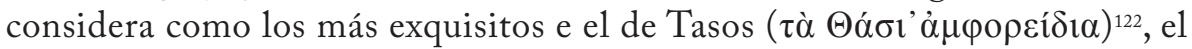
cual sobresale por su gran aroma y sabor, el famoso de Quíos (oĩvo $\delta \dot{\varepsilon}$ Xĩo

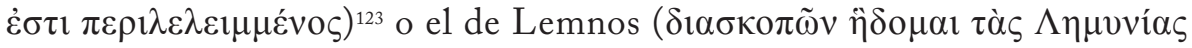
$\dot{\alpha} \mu \pi \dot{\varepsilon} \lambda \mathrm{ov \varsigma})^{124}$.

${ }^{116}$ Ec. 469; V. 306; 612; Ra. 376; Eq. 817; Av. 655, 788.

${ }_{117}$ Ec.651; 674; 694; 856; 1128; Pl. 595; V. 60; 311; 1401; 1005; Ach. 1096; 1138; 1142. Cf.

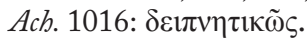

${ }^{118}$ Ec. 842-848.

${ }_{119}$ Pax 770; Lys. 1225; V, 1116. Cf. Ach. 227; Cf. García 2001: 33-35.

${ }^{120}$ Lys. 1227; V. 21; 1346; 1369.

${ }^{121} \mathrm{Nu} .50$.

${ }^{122}$ Ec. 1119. Cf. García 2001: 301; Salviat, 1986: 145-195.

${ }^{123}$ Ec. 1139. Cf. García 2001: 302.

${ }^{124}$ Pax 1162. Cf. García 2001: 302-303; Dalby 1995: 397-406; García (2002): 49-64. 
El simposio se inauguraba con una libación $(\sigma \pi \varepsilon \dot{\varepsilon} v \delta \omega)^{125}$ de vino puro a

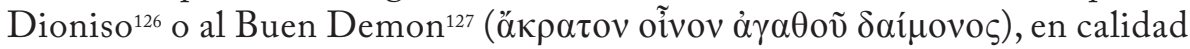
de acción de gracias ${ }^{128}$ :

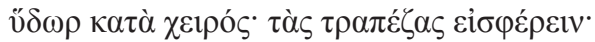

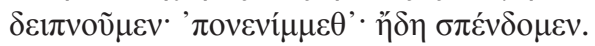

"Agua para las manos; trae las mesas, cenemos, bien lavados y ahora hagamos una libación".

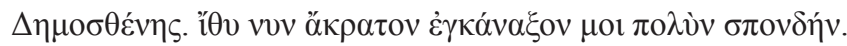

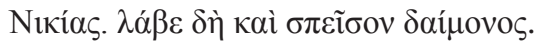

Demóstenes. "Venga, échame una gran libación de vino puro."

Nicias. ."Toma y liba en honor del Buen Demon"129.

A continuación, el simposiarca o anfitrión fijaba las medidas de la mezcla del agua y del vino en la crátera. Aristófanes propone las siguientes proporciones $^{130}$ :

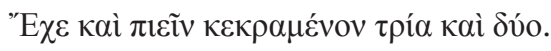

"Toma también para beber esta mezcla de tres partes de agua y dos de vino".

Pues el vino puro era propio de bárbaros o de borrachos empedernidos:

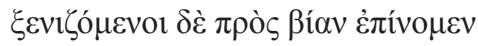

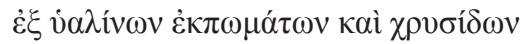

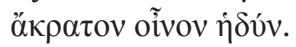

"Hospedados, a la fuerza bebimos dulce vino puro

en copas de cristal y de oro"131.

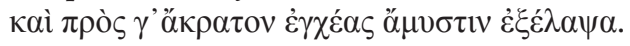

"Tras llenarlo de vino puro, me lo tomé de un trago"s32.

Para finalizar, no hay que excluir de este catálogo aristofánico ciertos neologismos inventados por nuestro cómico a partir de la unión de varios términos, con la clara intención de despertar la vis comica en el espectador,

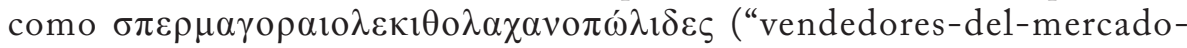

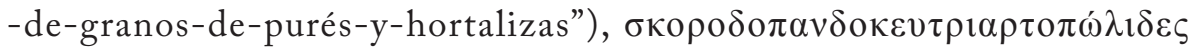

\footnotetext{
125 Ach. 199.

${ }^{126}$ P1. Smp., 176 a.

${ }^{127}$ Pax 300; Eq. 85. Cf. V. 524.

${ }^{128}$ V. $1216-1217$.

${ }^{129} \mathrm{Eq}$ 105-106.

${ }^{130}$ Eq. 1187.

${ }^{131}$ Ach. 75-76.

132 Ach. 1229.
} 
("hospederas-y-vendedoras-de-ajo-y-de-pan) ${ }^{133}$, y el multisilábico que recoge todos los manjares que se van a consumir en un banquete ${ }^{134}, \lambda \mathrm{o} \pi \alpha \delta$ o $\tau \varepsilon \mu \alpha \chi 0 \sigma$

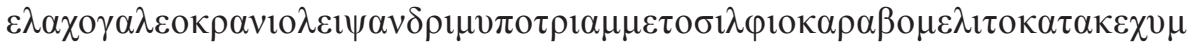

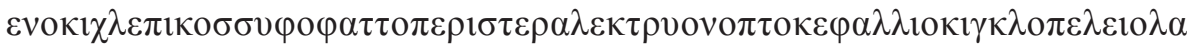
$\gamma \omega о \sigma ı \rho \iota \_\beta \alpha \varphi \eta \tau \rho \alpha \gamma \alpha v o \pi \tau \varepsilon \rho v \gamma \omega ́ v$ ("cazuela de pesca en rodajas-raya-cazón-trocitos de cabezas de pescado-con salsa picante-sazonados con silfio, miel y aceite-tordos sobre mirlos-palominos-torcaces-palomas-gallo-alondras asadas-chochas-pichones-liebres cocidas en vino-alas con sus ternillas").

La comedia aristofánica ofrece un material muy útil para conocer de cerca la gastronomía de la Grecia del siglo V a. C., no sólo desde el punto de vista de las descripciones sino también a través del léxico. En este género teatral, referente perfecto de la vida cotidiana y el carácter popular, la comida y el vino actúan como un personaje más, un protagonista primordial de las fiestas y de los banquetes, que tiene el don de congregar a las personas alrededor de la mesa al final de las representaciones.

\section{Bibliografía}

Amouretti, M. C. (1986), Le pain et le huile dans la Grèce antique, Annales littérein de l'Université de Besançon, 328, París.

Berthiaume, G. (1982), Les rôles de mágeiros. Étude sur la boucherie, la cuisine et le sacrifice dans la Grèce ancienne, Brill, Leiden.

Blumfield, A., (1997), "Cakes in the Liknon: Votives from the Sanctuary of Demeter and Kore on Acrocorinth", Hesperia 66.1: 147-172.

Bonacelli, B. (1924), Il silfio della'antica Cirenaica, Rassegnadella Colonia italiana, 22, Roma.

Bowie, E. L. (1995), "Wine in Old Comedy", Murray, O.-Tecusan. M. (eds.), In vino veritas, The British School al Rome, Londres, 113-125.

Braun, T. (1995), "Barley Cakes and Emmer Bread”, J. Wilkins-D. Harvey-M- Dobson (eds.), Food in Antiquity, University of Exeter Press, Exeter, 29-30.

Casevitz, M. (1990), "Repas, festins et banquets: un peu d'histoire des mots grecs", Cabiers des études ancienne 24: 201-221.

Dalby, A. (1995), "Topikos oinos: the named wines of Old Comedy", Harvey, D.-Wilkins, J. (eds.), J. Wilkins-D. Harvey-M. Dobson (eds.), Food in Antiquity, University of Exeter Press, Exeter, 397-406.

Daremberg, Ch.-Saglio, E. (1962-3), Dictionnaire des antiquités grecques et romaines, Hachette, Graz (París 1877-1919).

Davidson, A. (19812) Mediterranean Seafood, Prospect Books, Londres.

\footnotetext{
${ }^{133}$ Lys. 456-459.

${ }^{134}$ Ec. 1151-1153.
} 
Dover, K. (1993), Aristophanes Frogs, Clarendon Press, Oxford.

Feuardent, R. (1948), "Réflexions relatives au silphion”, Revue Numismatique 11: 27-32.

García Soler, Ma. J. (1997), "El pescado en la comedia griega”, in López Eire, A. (ed.), Sociedad, politica y literatura. Comedia griega antigua. Actas del I Congreso Internacional, Universidad de Salamanca, Salamanca, 279-285.

García Soler, Ma. J. (2001), El arte de comer en la antigua Grecia, Biblioteca Nueva, Madrid.

García Soler, Ma . J. (2002), "Los vinos de la comedia griega", DOURO-Estudios E̋ Documentos VII (13): 49-64.

García Soler, Ma .J. (2007), “La salsa de la Guerra (Aristófanes, Paz 236-288), in J. Alonso

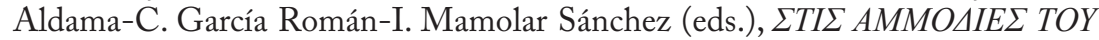
OMEHPOY. Homenaje a la Profesora Olga Omatos, Victoria-Gazteiz, 277-289.

García Soler, M. J. (2009), "La gastronomía como recurso cómico en la literatura griega”, in Ma. J. García Soler (ed.), El humor (y los humores) en el mundo antiguo, Amsterdam, 133-157.

García Soler,Ma .J.(2014), "Panes y pasteles en el ritual griego”, in A.Martínez Fernández-B. Ortega Villaro-H. Velasco López-H. Zamora Salmanca (eds.), Ágalma. Ofrenda desde Filología Clásica a Manuel García Teijeiro, Valladolid, 905-910.

Henderson, J. (1975), The Maculate Muse: Obscene language in Attic Comedy, Nueva York/ Londres.

de Hoz, J. (2011), Historia lingüistica de la península ibérica en la Antigüedad, CSIC, Madrid.

Jasny, N. (1950), "The daily bread of the ancient Greeks and Romans”, Osiris 9: 227-253.

Jay-Robert, G. (1998), “Symbolique de la nourriture et du repas chez Aristophane”, in P. Carmignani-J. Y. Laurichesse-J. Thomas (coords.), Saveurs, senteurs: Le goût de la Mediterranée. Actes du colloque. Université de Perpignan (13-15 novembre 1997), Perpignan, 23-39.

Moritz, L. A. (1958), Grains Mills and Flour in Classical Antiquity, Clarendon Press, Oxford.

Rainaud, A. (1911), “Silphium (Sílphion)”, DS, IV/2: 1337-1340.

Salviat, F. (1986), “Le vin des Thasos. Amphores, vin et sources écrites”, BCH supp. 13: 145-195.

Salza Prina Ricotti, E. (2005), L'arte del convito nella Grecia antica. La evoluzione del gusto da Achille ad Alessandro Magno, "L'Erma" di Bretschneider, Roma.

Stanford, W. B. (1963), Aristophanes. The Frogs, Bristol Classical Press, Londres.

Sterier, G. (1927), “Silphion”, RE, III A 1: cols. 103-114.

Taillardat, J. (1962), La image d'Aristophane. Études de langue et de style, París.

Thédenat, H. (1896), “Furnus”, DS II/2: 1420-1421.

Thiercy, P. (1997), "Le palais d'Aristophane ou les saveurs de la Polis", in P. Thiercy-M. Menu (eds.), Aristophane: La langue, la scène, la cité. Actes du colloque de Toulouse, 17-19 mars 1994, Bari, 131-177. 
Thiercy, P. (2003), “Cuisine et sexualité chez Aristophane”, Kentron 19:1-2: 17-30.

Wilkins, J. (1993), "The significance of food and eating in Greek comedy", Liverpool Classical Monthly 18: 66-74.

Wilkins, J. (1996), "Eating in Athenian comedy", in J. Wilkins (ed.), Food in European Literature, University of Exeter Press, Exeter, 46-56.

Wilkins, J., (2000) The Boastful Chef. The Discourse of Food In Ancient Greek Comedy, Oxford University Press, Oxford. 